\title{
Uterine Corpus Leiomyosarcoma and Endometrial Stromal Sarcoma pM1 TNM Finding v8
}

National Cancer Institute

\section{Source}

National Cancer Institute. Uterine Corpus Leiomyosarcoma and Endometrial Stromal

Sarcoma pM1 TNM Finding v8. NCI Thesaurus. Code C139823.

Uterine corpus leiomyosarcoma or endometrial stromal sarcoma with distant metastasis

(excluding adnexa, pelvic, and abdominal tissues). (from AJCC 8th Ed.) 